\title{
Nuclear Medicine Imaging in Epilepsy
}

\section{Jiaqiong Wang and Robert Carroll*}

Quantitative Imaging, Inc., Florida, USA

\begin{abstract}
For epilepsy patients who are not responding to anticonvulsant medications, surgery is an alternative treatment. A key issue in epilepsy surgery is the accurate localization of the "epileptogenic zone". To date, pre-surgical evaluation of the epileptogenic network can be carried out by electroencephalogram (EEG), video-EEG, magnetic resonance imaging, magneto-encephalography, single-photon emission computed tomography (SPECT) and positron emission tomography (PET). The interictal FDG-PET reveals hypometaboism at the epileptic focus, and it has been demonstrated to be much more sensitive than the interictal SPECT, and similarly sensitive to the ictal SPECT for the accurate localization of epileptogenic foci prior to surgical therapy. Visual assessment of 18F-FDG-PET is associated with interobserver and intraobserver variability. We have studied the issues of rigorous quantitation of FDG-PET brain studies and developed self-normalization technique. In addition to FDG-PET imaging, PET receptor imaging has also been demonstrated to provide significant insight into the mechanisms of neurotransmitters in epileptogenesis. In conclusion, we believe that nuclear medicine imaging can facilitate the identification of epileptic foci and investigate novel treatment for epilepsy.
\end{abstract}

Keywords: Epilepsy; Nuclear medicine; FDG-PET; Selfnormalization; Receptor imaging

\section{Introduction}

The majority of the treatments for epilepsy are anticonvulsant drugs. These medications include carbamazepine, diazepam, ethosucimide, gabapentin, levetiracetam, phenobartital, phenytoin, etc. Early identification of epilepsy non-responders allows prompt switching to an alternative therapy, as well as saving significant time and money and increasing patient and physician satisfaction. Clinicians must use sensitive and specific measurements to decide what works in individual patients.

For those patients whose seizures are not controlled by medications, re-evaluation is necessary and surgery is an alternative treatment for epilepsy. A key issue in epilepsy surgery is the accurate localization of the "epileptogenic zone (EZ)". However, the EZ in human epilepsy is not limited to one discrete focal brain area, but related to the activity of dynamic epileptogenic cortical networks [1]. To date, presurgical evaluation of the epileptogenic network can be performed by electroencephalogram (EEG), video-EEG, magnetic resonance imaging (MRI), magneto-encephalography, single-photon emission computed tomography (SPECT) and positron emission tomography (PET).

Previously ${ }^{99 m} \mathrm{Tc}$-hexamethyl propylene amine oxime ${ }^{99 \mathrm{~m}} \mathrm{Tc}$ HMPAO) SPECT has been utilized to directly image the location of epilepsy [2]. At the ictal phase, ${ }^{99 \mathrm{~m} T c-H M P A O ~ S P E C T ~ s h o w s ~ t h e ~}$ increased rate of regional cerebral blood flow (rCBF) from the epileptic focus during the seizure [2]. At the interictal phase, the epileptic focus reveals decreased rate of rCBF. Differently from SPECT, positron emission tomography (PET) utilizes 18Fluoro-2-deoxyglucose (18F-FDG) to measure glucose metabolism. The interictal FDGPET reveals hypometaboism at the epileptic focus. It has been demonstrated that the interictal FDG-PET is much more sensitive than the interictal SPECT, and similarly sensitive to the ictal SPECT for the accurate localization of epileptogenic foci prior to surgical therapy [3].

Visual assessment of 18F-FDG-PET is associated with very significant interobserver and intraobserver variability. To resolve this problem, we have studied the issues of rigorous quantitation of FDG brain PET studies [4,5]. Recently we have developed self-normalization of FDG PET-CT scans using the normal intact portions of that patient's brain, allowing for precise quantification of every area of the brain in relationship to the most normally functioning portions of that patient's brain. The brain area that is between the hypometabolic and hypermetabolic spectrum is considered normal for that patient at that time point. We have devised computer programs to normalized quantification based on the normal portions of a patient's brain. Selfnormalization identifies the least metabolically disturbed areas of the patient brain by excluding hypometabolic and hypermetabolic areas from the calculation of the mean, and then the entire data set is rescaled based upon normal for that patient. Self-normalization has been covered by two issued US patents by Robert G Carroll assigned to Quantitative Imaging Inc.: US patent $8,938,102$ (issued on Jan 20, 2015) [6] and US patent 9,025,845 (issued on May 5, 2015) [7]. This revolutionary self-normalization technique avoids the pitfalls of "normal" age matched control groups who by definition have not had the patient's experiential background, and greatly increases intercomparability, reproducibility and sensitivity in the measurement. In addition to our self-normalization approach, Peter and colleagues (2015) have developed global quantitative analysis technique to evaluate temporal lobe epilepsy, and showed that it can more accurately detect seizure lateralization and the potential therapeutic effect that can improve patient's quality of life [8].

Besides FDG-PET imaging, PET receptor imaging has also been demonstrated to provide significant insight into the mechanism of neurotransmitters in epileptogenesis. Pathophysiologically, different kinds of neurotransmitters and receptor subtypes are associated with epilepsy, including acetylcholine, adenosine, dopamine, gammaaminobutyric acid (GABA), glutamate, opiates, and serotonin. Various types of radiopharmaceuticals have been developed targeting to

*Corresponding author: Robert Carroll, M.D., Quantitative Imaging, Inc., Florida, USA, Tel: 33773-4411; E-mail: rbrtcrrll224@gmail.com

Received December 14, 2015; Accepted December 14, 2015; Published December 21, 2015

Citation: Wang J, Carroll R (2015) Nuclear Medicine Imaging in Epilepsy. J Epilepsy 2: e105. doi:10.4172/2472-0895.1000e105

Copyright: (C) 2015 Wang J, et al. This is an open-access article distributed under the terms of the Creative Commons Attribution License, which permits unrestricted use, distribution, and reproduction in any medium, provided the original author and source are credited. 
specific receptors. 11C-flumazenil can bind specifically to GABAAcentral benzodiazepine receptor. An 11C-flumazenil PET study revealed that regions of cortex with a normal MRI appearance from epilepsy patients showed abnormally increased binding of 11C-flumazenil $[9,10]$. 2'-Methoxyphenyl-(N-2'-pyridinyl)-p-18Ffluoro-benzamidoethylpiperazine (18F-MPPF) is a specific 5 -HT1A serotonin antagonist [11]. Didelot and colleagues (2010) utilized asymmetry index analysis of 18F-MPPF PET, and demonstrated that it was more sensitive and specific to localize the epileptogenic foci for pre-surgical assessment of patients with temporal lope epilepsies [12]. $11 \mathrm{C}$-cerfentanil is a mu opiate agonist receptor radioligand [13], it has been shown that there was increased binding of $11 \mathrm{C}$-cerfentanil in the temporal neocortex of unilateral temporal lobe epilepsy patients. Similarly, N1'-([11C] Methyl) naltrindole ([ $11 \mathrm{C}]-\mathrm{MeNTI})$ is a specific delta opiate receptor antagonist, and its binding also increased in the temporal cortex ipsilateral to the epilepsy focus [14].

In addition to PET receptor imaging, nuclear medicine imaging has also been utilized to investigate other mechanisms in epileptogenesis. Recently, Zhang and colleagues (2015) have utilized Indium-111-bis5-hydroxytryptamide-diethylenetriaminepentaacetate to image brain matrix metalloproteinase (MPO) activity by SPECT, and demonstrated the upregulation of MPO activity during epileptogenesis. This animal experiment result was consistent with the positive MPO immunostaining in the resected temporal lobe tissue from a patient with refractory epilepsy [15].

In summary, nuclear medicine imaging can promote our understanding of the mechanisms involved in epileptogenesis, facilitate the identification of epileptic foci and investigate novel treatment approaches for epilepsy.

\section{References}

1. Dixit AB, Banerjee J, Tripathi M, Chandra PS (2015) Presurgical epileptogenic network analysis: A way to enhance epilepsy surgery outcome. Neurol India 63: 743-750.
2. Shorvon SD (2009) A history of neuroimaging in epilepsy 1909-2009. Epilepsia 50: 39-49.

3. Sarikaya I (2015) PET studies in epilepsy. Am J Nucl Med Mol Imaging 5: 416-430.

4. Carroll R, Hipskind G (2010) Quantitative Assessment of Glucose Metabolism in 83 Brain Structures of Eighteen MVA Patients. Eighth World Congress on Brain Injury, Washington, DC, March 10-14.

5. Carroll RG, Briggs JJ (2012) Recovery from Fully Disabling Traumatic Brain Injury, 15 Year Followup. J Neurotrauma. 29: A-1-A-230 A189.

6. US Patent Issued to Quantitative Imaging on Jan. 20 for "Methods and Apparatus for Assessing Activity of an Organ and Uses Thereof".

7. US Patent Issued to Quantitative Imaging on May 5 for "Methods and Apparatus for Assessing Activity of an Organ and Uses Thereof".

8. Peter J, Houshmand S, Werner TJ, Rubello D, Alavi A (2015) Applications of global quantitative 18F-FDG-PET analysis in temporal lobe epilepsy. Nucl Med Commun.

9. Richardson MP, Koepp MJ, Brooks DJ, Fish DR, Duncan JS (1996) Benzodiazepine receptors in focal epilepsy with cortical dysgenesis: an 11C-flumazenil PET study. Ann Neurol 40: 188-198.

10. Hammers A, Koepp MJ, Hurlemann R, Thom M, Richardson MP, et al. (2002) Abnormalities of grey and white matter [11C] flumazenil binding in tempora lobe epilepsy with normal MRI. Brain 125: 2257-2271.

11. Costes N, Merlet I, Ostrowsky K, Faillenot I, Lavenne F, et al. (2005) A 18F-MPPF PET normative database of 5-HT1A receptor binding in men and women over aging. J Nucl Med 46: 1980-1989.

12. Didelot A, Mauguière F, Redouté J, Bouvard S, Lothe A, et al. (2010) Voxel-based analysis of asymmetry index maps increases the specificity of 18F-MPPF PET abnormalities for localizing the epileptogenic zone in temporal lobe epilepsies. J Nucl Med 51: 1732-1739.

13. Mayberg HS, Sadzot B, Meltzer CC, Fisher RS, Lesser RP, et al. (1991) Quantification of mu and non-mu opiate receptors in temporal lobe epilepsy using positron emission tomography. Ann Neurol 30: 3-11.

14. Madar I, Lesser RP, Krauss G, Zubieta JK, Lever JR, et al. (1997) Imaging of delta- and mu-opioid receptors in temporal lobe epilepsy by positron emission tomography. Ann Neurol 41: 358-367.

15. Zhang Y, Seeburg DP, Pulli B, Wojtkiewicz GR, Bure L, et al (2015) Radiology. Myeloperoxidase Nuclear Imaging for Epileptogenesis 23: 141922. 